\title{
TRANSITION TIME ANALYSIS IN SINGULARLY PERTURBED BOUNDARY VALUE PROBLEMS
}

\author{
FREDDY DUMORTIER AND BERT SMITS
}

\begin{abstract}
The paper deals with the boundary value problem $\varepsilon \ddot{x}+x \dot{x}-x^{2}=0$, with $x(0)=A, x(T)=B$ for $A, B, T>0$ and $\varepsilon>0$ close to zero. It is shown that for $T$ sufficiently big, the problem has exactly three solutions, two of which reach negative values. Solutions reaching negative values occur for $T \geq T(\varepsilon)>0$ and we show that asymptotically for $\varepsilon \rightarrow 0, T(\varepsilon) \sim-\ln (\varepsilon)$, i.e. $\lim _{\varepsilon \rightarrow 0}-\frac{T(\varepsilon)}{\ln (\varepsilon)}=1$. The main tools are transit time analysis in the Lienard plane and normal form techniques. As such the methods are rather qualitative and useful in other similar problems.
\end{abstract}

\section{INTRODUCTION}

In this paper, the authors will apply desingularization techniques to a singularly perturbed boundary value problem. By doing so, we will be able to explain apparent transition phenomena in a geometric way.

The equation we study is given by

$$
\varepsilon \ddot{x}+x \dot{x}-x^{2}=0
$$

and was introduced by O'Malley [AO]. The geometric approach consists of rewriting (1) as a vector field in the Lienard plane and then to study the phase portrait. It turns out that the global desingularization technique reveals essential information about the flow near the degenerate singularity at the origin.

O'Malley's interest included the non-uniqueness of solutions to the boundary value problem (1) with $x(0)=A, x(T)=B$, for $A, B, T>0$. We will prove the existence of exactly three such solutions, provided $T$ is large enough, with the aid of transition time analysis as in [CD]. In Figure 1 we show three such solutions for $\varepsilon=1, T=4.3, A=B=10$.

Furthermore, we will give an asymptotic estimate for the minimal transition time as $\varepsilon \rightarrow 0$ using normal forms for the blown-up vector field.

We also prove that outer expansion techniques are equivalent to center manifold reduction and shed light on a good choice of initial conditions.

Received by the editors May 18, 1994 and, in revised form, November 29, 1994.

1991 Mathematics Subject Classification. Primary 34B15, 34C20, 34E15, 34C20, 58F36; Secondary 34E05, 58F14.

Key words and phrases. Global blowing up, transition time, asymptotic analysis, center manifold. 

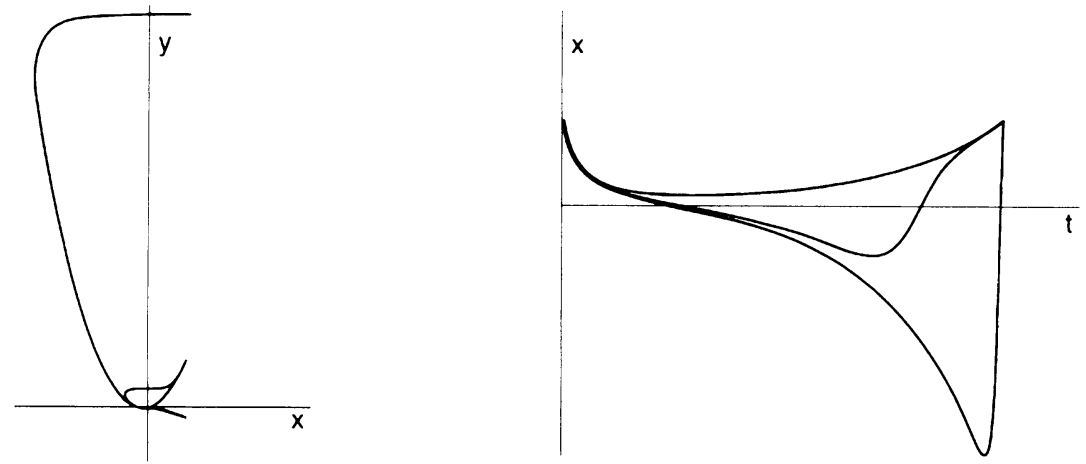

FIGURE 1. Three different solutions to the same boundary value problem.

\section{Setup}

Rewriting (1) as a Liénard vector field is a standard method which applies to all perturbation problems of the form

$$
\varepsilon \ddot{x}+f(x) \dot{x}+g(x)=0 .
$$

Put $y=\varepsilon \dot{x}+F(x)$ where

$$
F(x)=\int_{0}^{x} f(u) d u .
$$

This last equation is the so called "Liénard transformation". After rescaling time by a factor $1 / \varepsilon$ and renaming the variables we obtain

$$
\left\{\begin{array}{l}
\dot{x}=y-F(x) \\
\dot{y}=-\varepsilon g(x) .
\end{array}\right.
$$

For our equation this yields

$$
\left\{\begin{array}{l}
\dot{x}=y-\frac{x^{2}}{2} \\
\dot{y}=\varepsilon x^{2}
\end{array}\right.
$$

hence a one parameter family $\left\{X_{\varepsilon}=X(x, y, \varepsilon)\right\}_{\varepsilon>0}$. Later on, we will have to perform a desingularization because of the appearance of a degenerate singularity at the origin. The unperturbed system $\varepsilon=0$, which has has a parabola of singularities, all but one of which are elementary (i.e., having a linear part with one non-zero eigenvalue), is drawn in Figure 2.

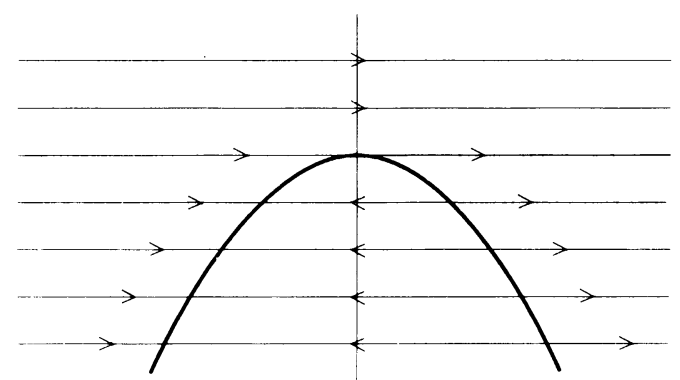

FIGURE 2. Phase portrait of the unperturbed system. 
The desingularization at $(0,0)$ will consist of a weighted blow-up. By doing this, we lose the family character of $X(x, y, \varepsilon)$ and the resulting object need not be a vector field any more, but only a "foliated local vector field". A formal build up of this procedure has been done in [DeR] and in [DR]. For a didactic introduction, the reader can look in [D], where the Andronov-Hopf bifurcation is discussed as an example. We limit ourselves here to describing the desingularizing maps.

\section{TRANSITION TIME ANALYSIS OF A SINGLE VECTOR FIELD}

We first observe that, fixing $\varepsilon$, we can rescale the original Liénard system by taking

$$
\left\{\begin{aligned}
x & =\varepsilon \bar{x} \\
y & =\varepsilon^{2} \bar{y} \\
\varepsilon t & =\bar{t}
\end{aligned}\right.
$$

to obtain

$$
\tilde{X}:\left\{\begin{array}{l}
\dot{\bar{x}}=\bar{y}-\frac{\bar{x}^{2}}{2} \\
\dot{\bar{y}}=\bar{x}^{2}
\end{array}\right.
$$

(See Figure 3.)

This proves that all vector fields in the family $\left\{X_{\varepsilon}\right\}_{\varepsilon>0}$ are linearly equivalent to $\tilde{X}$. We would like to stress, however, that it is purely coincidental and not necessary for the desingularization analysis in the sequel to have $\tilde{X}$ independent of $\varepsilon$. Let us for the rest of this paragraph concentrate on $\tilde{X}$. For each fixed $\varepsilon$, the original boundary value problem

$$
x(0)=A, \quad x(T)=B, \text { with } A, B>0
$$

is transformed into

$$
\bar{x}(0)=\bar{A}, \bar{x}(\bar{T})=\bar{B} \text {, with } \bar{T}=T, \bar{A}=A / \varepsilon, \bar{B}=B / \varepsilon .
$$

Let us in the sequel omit the bars over $x, y, A, B$ and $T$. The singularity at the origin can be identified topologically as a cusp, by means of a quasihomogeneous blow-up (see [D, p. 21] for the calculation). All the singularities on the blow-up locus are hyperbolic. It was then proved in [CD] that the original transition time goes monotonously to infinity as the trajectory approaches the singularity. This implies that solutions obtained from a movement along the

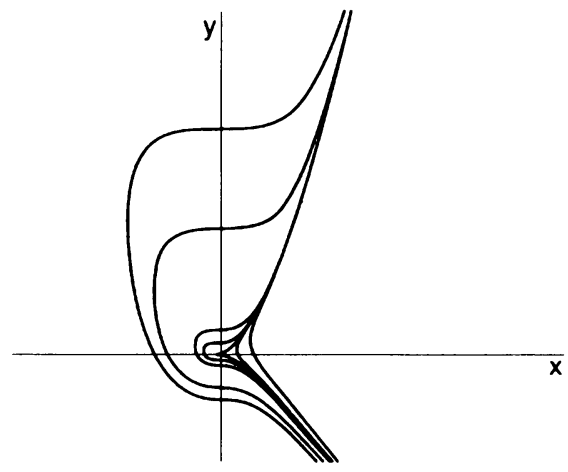

FIGURE 3. Phase portrait of $X(x, y, \varepsilon)$ for $\varepsilon>0$. 
right side of the singularity are unique per given $T$, if $T$ is large enough. In a neighbourhood of the cusp we also have monotonicity of the time on the left side thus proving the existence of a second solution to the boundary value problem. The third and final solution to the boundary value problem can be found in trajectories that move near the left branch of the parabola $y=\frac{x^{2}}{2}$ for a long time before crossing over to the right. To prove uniqueness in this case, we will rescale the vector field $\tilde{X}$ to get an expression at infinity and use monotonicity of the time map along some saddle sector at infinity. This involves some careful treatment because we are again considering a different (scaled) time map in the neighbourhood of a semi-hyperbolic singularity.

The proposed rescaling at infinity means transforming (3) with

$$
\left\{\begin{array}{l}
x=u / s \\
y=1 / s^{2}
\end{array}\right.
$$

This is called a "quasi-homogeneous blow up at infinity (on the Poincaré sphere) in the $y$-direction". It yields a vector field

$$
X^{\prime}:\left\{\begin{array}{l}
\dot{s}=-\frac{1}{2} s u^{2} \\
\dot{u}=\frac{1}{s}-\frac{u^{2}}{2 s}-\frac{1}{2} u^{3}
\end{array}\right.
$$

and by multiplying the time with a factor $s$ we get

$$
\bar{X}^{\prime}:\left\{\begin{array}{l}
\dot{s}=-\frac{1}{2} s^{2} u^{2} \\
\dot{u}=1-\frac{u^{2}}{2}-\frac{s u^{3}}{2} .
\end{array}\right.
$$

On the blow-up locus $\{s=0\}$, representing infinity on the Poincaré sphere, we find two singularities $k_{1}$ and $k_{2}(s=0, u= \pm \sqrt{2})$ that are semi-hyperbolic:

$$
\begin{aligned}
& D \bar{X}_{(0,+\sqrt{2})}^{\prime}=\left(\begin{array}{cc}
0 & 0 \\
-\sqrt{2} & -\sqrt{2}
\end{array}\right), \\
& D \bar{X}_{(0,-\sqrt{2})}^{\prime}=\left(\begin{array}{cc}
0 & 0 \\
+\sqrt{2} & +\sqrt{2}
\end{array}\right) .
\end{aligned}
$$

To explain the behaviour in the $s$-direction we perform a center manifold reduction [Ca] at $k_{1}$ and $k_{2}$. At $k_{1}=(0,-\sqrt{2})$, we simplify $\bar{X}^{\prime}$ by substituting $w=u+\sqrt{2}$ :

$$
\left\{\begin{array}{l}
\dot{s}=-\frac{1}{2} s^{2}(w-\sqrt{2})^{2} \\
\dot{w}=1-\frac{1}{2}(w-\sqrt{2})^{2}-\frac{1}{2} s(w-\sqrt{2})^{3} .
\end{array}\right.
$$

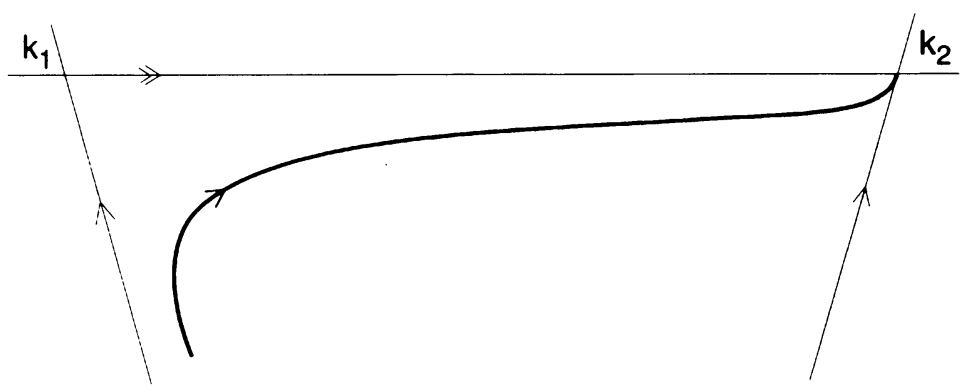

FIgURE 4. Partial phase portrait at infinity. 
Writing the center manifold as a graph $(s, w(s))$ and using the invariance under the flow we find

$$
w(s)=\frac{1}{\sqrt{2}} s+O\left(s^{2}\right)
$$

which results in the quadratic behaviour

$$
\dot{s}=-s^{2}+O\left(s^{3}\right)
$$

along the center manifold. We conclude that $k_{1}$ is a saddle (for $\varepsilon>0$ ). At $k_{2}=(0,+\sqrt{2})$ we put $w=u+\sqrt{2}$ :

$$
\left\{\begin{array}{l}
\dot{s}=-\frac{1}{2} s^{2}(w+\sqrt{2})^{2} \\
\dot{w}=1-\frac{1}{2}(w+\sqrt{2})^{2}-\frac{1}{2} s(w+\sqrt{2})^{3}
\end{array}\right.
$$

and in a similar way we see that $k_{2}$ is a sink (Figure 4). As we indicated before, it is not enough to prove monotonicity of the new time, because we have to consider the original $\tilde{X}$-time that we have altered in the rescaling. We will do this by using appropriate normal forms. By using a theorem of Takens [T1], the vector field $\bar{X}^{\prime}$ near $k_{1}$ is $C^{r}$-conjugate (for any $r$ ) to some

$$
\bar{X}^{\prime \prime}:\left\{\begin{array}{l}
\dot{x}=f(x) \\
\dot{y}=g(x) y
\end{array}\right.
$$

and we may suppose that the conjugacy sends $\{s=0\}$ to $\{x=0\}$. In our case, we have $g(0) \neq 0, f(0)=f^{\prime}(0)=0, f^{\prime \prime}(0) \neq 0$. Hence, we can rewrite $\bar{X}^{\prime \prime}$ as

$$
\bar{X}^{\prime \prime}:\left\{\begin{array}{l}
\dot{x}=x^{2} \bar{f}(x) \\
\dot{y}=h(x) y \bar{f}(x)
\end{array}\right.
$$

with $\bar{f}(0) \neq 0$ and $h(0) \neq 0$. Dividing by $\bar{f}(x)$ we get a simpler equivalent vector field

$$
X^{S}:\left\{\begin{array}{l}
\dot{x}=x^{2} \\
\dot{y}=-h(x) y .
\end{array}\right.
$$

Recall that, to obtain the original vector field again, we have to divide $X^{S}$ by the function $s / \bar{f}(x)$, which is given by some $x H(x, y)$ with $H(0,0) \neq 0$. We restrict $H$ to some compact neighbourhood of the singularity and suppose, without loss of generality, that it contains $[0,1]^{2}$. We now prove the following Proposition. Let $X=\frac{1}{x^{k} H(x, y)} X^{S}$ with $X^{S}=x^{n} \frac{\partial}{\partial x}-h(x) y \frac{\partial}{\partial y}, n>k$ on $\{x>0\}$ and $H, h C^{r}$-functions $(r \geq 2)$, with $H(0,0)>0, h(0)>0$. Let $C_{1}$ and $C_{2}$ be two transversal sections to the positive $y$-axis and the positive $x$-axis respectively. Let $s$ be a regular parameter on $C_{1}$. Then the transit time from $C_{1}$ to $C_{2}$ expressed in $s$ goes monotonously to infinity with derivative going to $-\infty$.

Proof. We first calculate the time between the two standard sections $\{x=1\}$ and $\{y=\delta\}$ for some $\delta>0$ in the normal form and use $s$ to be $x_{0}$ for any point $\left(x_{0}, \delta\right)$ (see Figure 5).

If $\delta=1$, the time map is given by

$$
T\left(x_{0}\right)=\int_{x=x_{0}}^{1} H\left(x, y^{x_{0}}(x)\right) \frac{d x}{x^{n-k}}
$$




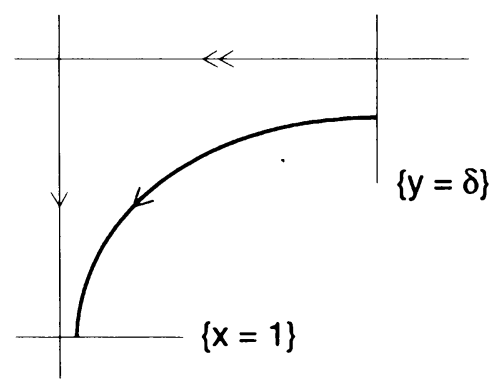

Figure 5. Transit from $\{y=\delta\}$ to $\{x=1\}$.

and its derivative is

$$
\frac{\partial T}{\partial x_{0}}\left(x_{0}\right)=-\frac{H\left(x_{0}, 1\right)}{x_{0}^{n-k}}+\int_{x=x_{0}}^{1} \frac{\partial G}{\partial x_{0}}\left(x, x_{0}\right) \frac{d x}{x^{n-k}}
$$

with $G\left(x, x_{0}\right)=H\left(x, y^{x_{0}}(x)\right)$. We now give an absolute estimate for the integral on the right-hand side of (6). If we suppose that $\left|\frac{\partial H}{\partial y}(x, y)\right| \leq M$ on $[0,1]^{2}$ then we get

$$
\begin{aligned}
\left|\int_{x=x_{0}}^{1} \frac{\partial G}{\partial x_{0}}\left(x, x_{0}\right) \frac{d x}{x^{n-k}}\right| & =\left|\int_{x=x_{0}}^{1} \frac{\partial H}{\partial y}\left(x, y^{x_{0}}(x)\right) \frac{\partial y^{x_{0}}(x)}{\partial x_{0}} \frac{d x}{x^{n-k}}\right| \\
& \leq M \int_{x=x_{0}}^{1}\left|\frac{\partial y^{x_{0}}(x)}{\partial x_{0}}\right| \frac{d x}{x^{n-k}} .
\end{aligned}
$$

If we change $(x, y)$ by $(x, \bar{y})$ with $y=\delta \bar{y}$ then the expression of $X^{S}$ does not change while $X$ changes into $\frac{1}{x^{k} H(x, \delta \bar{y})} X^{S}$, which we write as $\frac{1}{x^{k} \tilde{H}(x, \bar{y})} X^{S}$. On the other hand, $\frac{\partial H}{\bar{y}}=\delta \frac{\partial H}{y} \leq K \delta$, for some $K$ independent of $\delta$ if we take $(x, \bar{y}) \in[0,1]^{2}$ and hence $(x, y) \in[0,1] \times[0, \delta]$. As such in (7) we may suppose that $M=\delta K$, with $\delta$ as small as we want. In the original $(x, y)$-coordinates this corresponds to studying the transit time from $\{y=\delta\}$ to $\{x=1\}$. As in the end we will fix some $\delta>0$, this extra assumption on $M$ is permitted. Let us continue to work in $(x, \bar{y})$-coordinates from $\{\bar{y}=1\}$ to $\{x=1\}$. Now

$$
\frac{d \bar{y}}{d x}=\frac{-h(x) \bar{y}}{x^{n}} \Rightarrow \bar{y}(x)=e^{-\int_{x_{0}}^{x} \frac{h(u)}{u^{n}} d u}
$$

and thus

$$
\left|\frac{\partial \bar{y}^{x_{0}}(x)}{\partial x_{0}}\right|=\frac{h\left(x_{0}\right)}{x_{0}^{n}} e^{-\int_{x_{0}}^{x} \frac{h(u)}{u^{n}} d u} \leq \frac{C^{\prime}}{x_{0}^{n}} e^{-C\left(\frac{1}{x_{0}^{n-1}}-\frac{1}{x^{n-1}}\right)}
$$

for some $C>0, C^{\prime}>0$. This leaves us with finding an estimate for

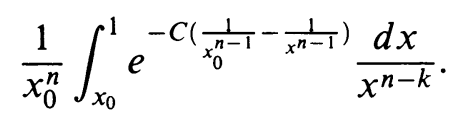

Changing over to the time variable again we get

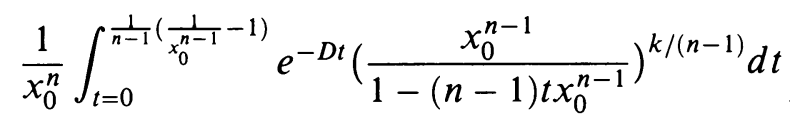


with $D=(n-1) C>0$, or

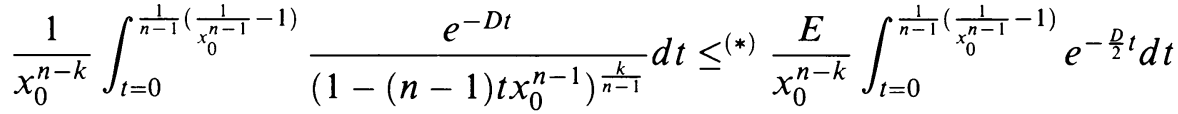

$$
\begin{aligned}
& =\frac{E}{x_{0}^{n-k}} \frac{2}{D}\left(1-e^{-\frac{D}{2(n-1)}\left(\frac{1}{\left.x_{0}^{n-1}-1\right)}\right)} \leq \frac{F}{x_{0}^{n-k}} .\right.
\end{aligned}
$$

In equality $(*)$ we use that

$$
e^{-\frac{D}{2} t}\left(1-(n-1) t x_{0}^{n-1}\right)^{-\frac{k}{n-1}} \leq E
$$

for all $t \in\left[0, \frac{1}{n-1}\left(\frac{1}{x_{0}^{n-1}}-1\right)\right]$ and for some $E>0$, only depending on $D$ and not on $x_{0}$, for $x_{0}$ sufficiently small. We therefore first observe that since $k \leq n-1,0<x_{0}<1$ and

$$
0<x_{0}^{n-1}<1-(n-1) t x_{0}^{n-1} \leq 1,
$$

we have

$$
\frac{e^{-\frac{D}{2} t}}{\left(1-(n-1) t x_{0}^{n-1}\right)^{\frac{k}{n-1}}} \leq \frac{e^{-\frac{D}{2} t}}{\left(1-(n-1) t x_{0}^{n-1}\right)}=\frac{e^{-u}}{1-\varepsilon u}
$$

with $u=\frac{D}{2} t \in\left[0, \frac{D}{2(n-1)}\left(\frac{1}{x_{0}^{n-1}}-1\right)\right]$ and $\varepsilon=\frac{2}{D}(n-1) x_{0}^{n-1}$. Inequality (8) follows if we can prove that there is some $E>0$ not depending on $\varepsilon$ (for $\varepsilon$ sufficiently small) such that

$$
\frac{e^{-u}}{1-\varepsilon u} \leq E
$$

for $u \in\left[0, \frac{1}{\varepsilon}-A\right]$ and $A>0$ some arbitrary but fixed real number. As $e^{-u}(1-\varepsilon u)^{-1}$ is monotone decreasing on $\left[0, \frac{1}{\varepsilon}-1\right]$, monotone increasing on $\left[\frac{1}{\varepsilon}-1, \frac{1}{\varepsilon}\left[\right.\right.$ and its value at $\frac{1}{\varepsilon}-A$ is $\frac{e^{-\left(A+\frac{1}{\varepsilon}\right)}}{A \varepsilon}$, inequality (9) is straightforward.

The final estimate can then be written as

$$
\left|\int_{x=x_{0}}^{1} \frac{\partial G}{\partial x_{0}}\left(x, x_{0}\right) \frac{d x}{x^{n-k}}\right| \leq \delta K C^{\prime} F \frac{1}{x_{0}^{n-k}} .
$$

Let $L$ be a lower bound for $H(x, y)$. Choosing $\delta<\frac{L}{2 K C^{\prime} F}$ we have proved that

$$
\frac{\partial T}{\partial x_{0}}\left(x_{0}\right) \rightarrow-\infty \quad \text { as } x_{0} \rightarrow 0
$$

and hence that $T\left(x_{0}\right) \rightarrow+\infty$ in a monotone way.

The fact that the monotonicity of the transit time is independent of the chosen sections is a consequence of the fact that a transit time between sections both transverse to the same separatrix has a bounded derivative. Along the $x$ axis this is a standard observation and along the $y$-axis it easily follows from the results in $[C D]$. The monotonicity is clearly not depending on the regular parameter.

The above theorem is sufficient to prove the existence of exactly three solutions to the boundary value problem for large enough $T$.

A transit time between sections $\left\{x=X_{0}\right\}$ and $\left\{y=Y_{0}\right\}$ in system (3) corresponds after blowing up to a movement between sections $\left\{u=X_{0} s\right\}$ and 

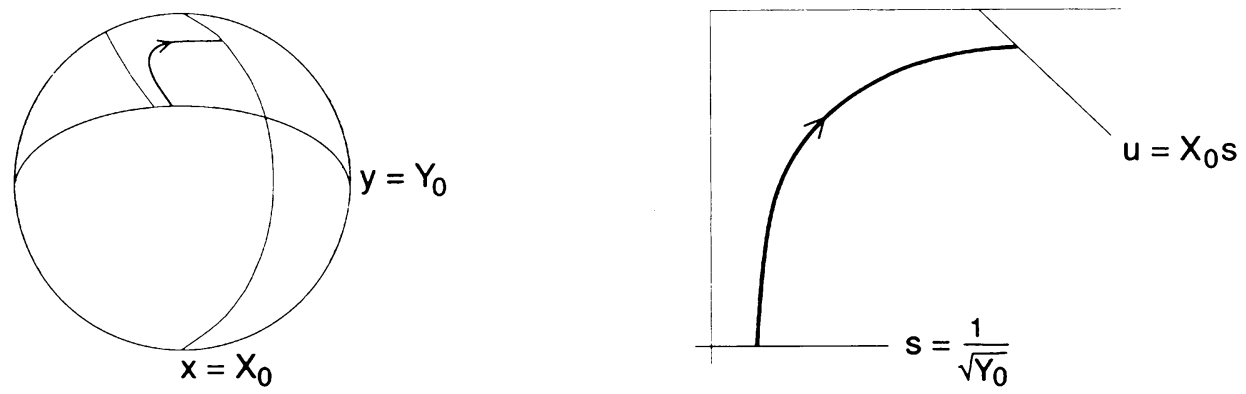

Figure 6. Saddle sectors on the Poincaré sphere and in the blown-up situation.

$\left\{s=\frac{1}{\sqrt{Y_{0}}}\right\}$ which, in normal form coordinates, transform to some transverse sections $\left\{u=s \psi_{1}(s)\right\}$ and $\left\{s=\psi_{2}(u)\right\}$, with $\psi_{i}(0) \neq 0, i=1,2$ (see Figure $6)$. We are now in a situation like in the proposition with $k=1$ and $n=2$, thus the transit time between these sections goes monotonously to infinity as the starting point gets closer to the separatrix.

From the implicit function theorem, it follows that the transition time is analytic, hence continuous. Therefore, there exists a minimal transition time for solutions passing left of the origin. We will use global desingularization to estimate its asymptotic behaviour as $\varepsilon \rightarrow 0$.

\section{Desingularization OF THE FAMILY OF VeCTOR FIELDS}

Consider the map

$$
\Phi: \mathbb{S}_{+}^{2} \times\left[0, U\left[\rightarrow \mathbb{R} \times \mathbb{R}^{2}:(\bar{x}, \bar{y}, \bar{\varepsilon}, u) \mapsto\left(u \bar{x}, u^{2} \bar{y}, u \bar{\varepsilon}\right)=(x, y, \varepsilon) .\right.\right.
$$

The pull back of $X$ by $\Phi$ results in a vector field $\hat{X}$ on $\mathbb{S}_{+}^{2} \times[0, U[$. Dividing $\hat{X}$ by the function $u$ we obtain an equivalent vector field $\bar{X}$, which is called the blown-up vector field, from which the local vector field will be constructed. In practice, we will use two systems of charts, covering the blow-up locus $\{u=0\}$.

3.1. Rescaling of the family. This is the traditional part of the blow-up method, as described in, e.g., [D] or [AP]. In (10), we take $\bar{\varepsilon}=1$ and let $(\bar{x}, \bar{y})$ vary in some large disk $\bar{D}$ of $\mathbb{R}^{2}$, the blown-up vector field is in these coordinates given by

$$
\bar{X}:\left\{\begin{array}{l}
\dot{\bar{x}}=\bar{y}-\frac{\bar{x}^{2}}{2} \\
\dot{\bar{y}}=\bar{x}^{2} .
\end{array}\right.
$$

Note that the vector field is described by the same equations as in (3), only the coordinate system is different. The singularity at $(0,0)$ has already been identified topologically as a cusp.

3.2. Phase directional rescaling. Looking at this chart is the really innovative part of the global blowing-up technique. In (10), we now choose $\bar{x}^{2}+\bar{y}^{2}=1$ and take $\bar{\varepsilon}$ in a neighbourhood of 0 . From the form of $\Phi$ we have $u \bar{\varepsilon}=\varepsilon$, hence $\dot{u} \bar{\varepsilon}+u \dot{\bar{\varepsilon}}=0$, or $\frac{\dot{\bar{\varepsilon}}}{\bar{\varepsilon}}=-\frac{\dot{u}}{u}$. To simplify the calculations, we will merely consider a directional blow-up, i.e., we look at four subcharts each covering an open half circle. For reasons of symmetry, it suffices to consider just two such 
subcharts. The actual rescaling formula (for the $\bar{x}$-direction) is given by

$$
\left\{\begin{array}{l}
x=u \\
y=u^{2} v \\
\varepsilon=u \bar{\varepsilon}
\end{array}\right.
$$

which transforms the vector field $X$ to

$$
\hat{U}:\left\{\begin{array}{l}
\dot{u}=u^{2}\left(v-\frac{1}{2}\right) \\
\dot{v}=u\left(\bar{\varepsilon}-2 v\left(v-\frac{1}{2}\right)\right) \\
\dot{\bar{\varepsilon}}=-u \bar{\varepsilon}\left(v-\frac{1}{2}\right) .
\end{array}\right.
$$

After division by $u$ we obtain

$$
\bar{U}=\frac{1}{u} \hat{U}:\left\{\begin{array}{l}
\dot{u}=u\left(v-\frac{1}{2}\right) \\
\dot{v}=\bar{\varepsilon}-2 v\left(v-\frac{1}{2}\right) \\
\dot{\bar{\varepsilon}}=-\bar{\varepsilon}\left(v-\frac{1}{2}\right) .
\end{array}\right.
$$

On the blow-up locus $\{u=0\}$ we notice two singularities for the unperturbed system at $v=0$ and $v=\frac{1}{2}$.

The singularity at $(0,0,0)$ is hyperbolic, for

$$
\left.D \bar{U}\right|_{(0,0,0)}=\left(\begin{array}{ccc}
-\frac{1}{2} & 0 & 0 \\
0 & +1 & 1 \\
0 & 0 & +\frac{1}{2}
\end{array}\right)
$$

which locally gives a phase portrait as in Figure 7 .

At $\left(0, \frac{1}{2}, 0\right)$ we find a semi-hyperbolic singularity:

$$
\left.D \bar{U}\right|_{\left(0, \frac{1}{2}, 0\right)}=\left(\begin{array}{ccc}
0 & 0 & 0 \\
0 & -1 & 1 \\
0 & 0 & 0
\end{array}\right) .
$$

Along the circle (represented by the $v$-direction), the movement will be attracting. To see what happens in the $\bar{\varepsilon}$-direction, we look at the 2-dimensional part of the vector field

$$
\left\{\begin{array}{l}
\dot{v}=\bar{\varepsilon}-2 v\left(v-\frac{1}{2}\right) \\
\dot{\bar{\varepsilon}}=-\bar{\varepsilon}\left(v-\frac{1}{2}\right)
\end{array}\right.
$$

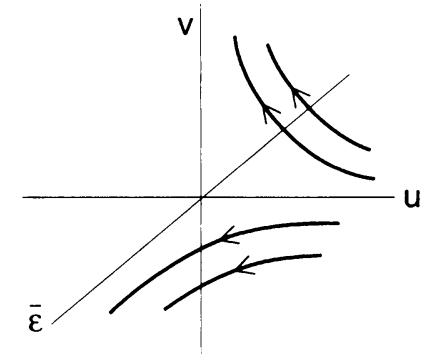

FIGURE 7. Local phase portrait around the hyperbolic singularity. 
and perform another center manifold reduction. To simplify the calculation, we again translate by $w=v-\frac{1}{2}$ and study

$$
\left\{\begin{array}{l}
\dot{w}=\bar{\varepsilon}-2 w\left(w+\frac{1}{2}\right) \\
\dot{\bar{\varepsilon}}=-\bar{\varepsilon} w
\end{array}\right.
$$

Writing an invariant center manifold as a graph $(\bar{\varepsilon}, w(\bar{\varepsilon}))$ with

$$
w(\bar{\varepsilon})=\alpha \bar{\varepsilon}+O\left(\bar{\varepsilon}^{2}\right) .
$$

Expressing the invariance under the flow we find $\alpha=1$, thus

$$
\dot{\bar{\varepsilon}}=-\bar{\varepsilon}^{2}+O\left(\bar{\varepsilon}^{3}\right)
$$

which means that the singularity has an attracting behaviour in the $\bar{\varepsilon}$-direction along the center manifold.

With the phase-directional rescaling in the $y$-direction,

$$
\left\{\begin{array}{l}
x=u v \\
y=u^{2} \\
\varepsilon=u \bar{\varepsilon}
\end{array}\right.
$$

and dividing by $u$ we analogously obtain

$$
\bar{U}^{\prime}:\left\{\begin{array}{l}
\dot{u}=\frac{1}{2} u \bar{\varepsilon} v^{2} \\
\dot{v}=1-\frac{v^{2}}{2}-\frac{\bar{\varepsilon} v^{3}}{2} \\
\dot{\bar{\varepsilon}}=-\frac{1}{2} \bar{\varepsilon}^{2} v^{2}
\end{array}\right.
$$

with singularities at $(0, \pm \sqrt{2}, 0)$ with respective Jacobian matrices

$$
\left.D \bar{U}^{\prime}\right|_{(0, \pm \sqrt{2}, 0)}=\left(\begin{array}{ccc}
0 & 0 & 0 \\
0 & \mp \sqrt{2} & \mp \sqrt{2} \\
0 & 0 & 0
\end{array}\right) .
$$

Again by center manifold reduction, we find that both singularities are attracting in the $\bar{\varepsilon}$-direction.

Glueing the charts together, we are now ready to draw the local phase portrait around $(0,0)$, as in Figure 8.

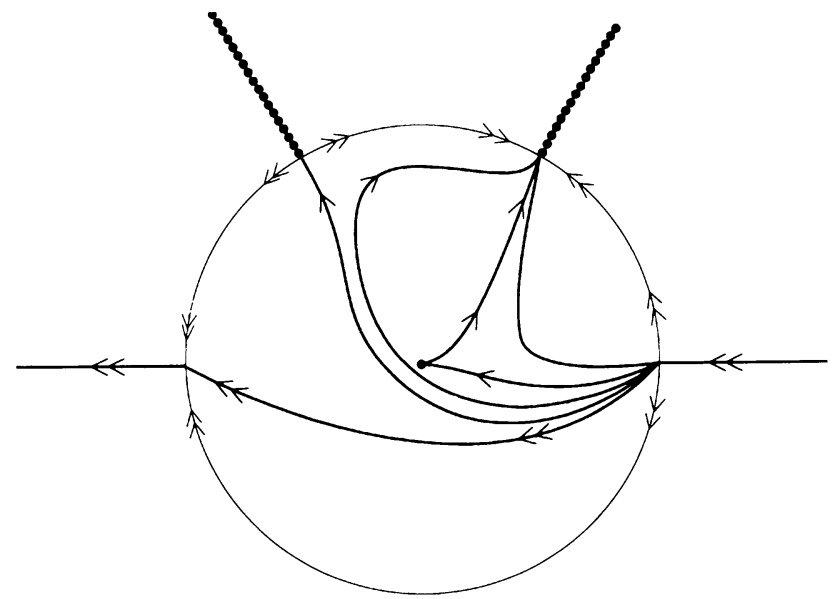

FIGURE 8. Global blow-up of the degenerate singularity. 


\section{ASYMPTOTIC ESTIMATES FOR THE TIME MAP AS $\varepsilon \rightarrow 0$}

We are now going to make use of the global blowing up method in determining the asymptotic behaviour of a minimal time solution. We will consider various passages in the neighbourhood of singularities by means of normal form theory, thus providing a useful tool for the calculation of transit time asymptotics in general. As indicated in the end of the previous paragraph, the global phase portrait at the origin contains five singularities: two hyperbolic ones, two semihyperbolic ones and a cusp singularity. Any solution to the boundary value problem must, for $\varepsilon$ sufficiently small, pass near the hyperbolic singularity to the right and near at least one of the two semi-hyperbolic ones. Hence, we will have to find lower estimates for the transit time in a neighbourhood of those singularities. First, we remark that a passage along the curve of zeroes outside a neighbourhood of the cusp has no big influence on the transit time asymptotics.

4.1. Passage along the parabola. By a theorem of Takens [T2] (or using the $\varepsilon$-dependent version in [B]) in a neighbourhood of a point $\left(x_{0}, x_{0}^{2} / 2\right) \neq(0,0)$ on the parabola of zeroes, the vector field is $C^{r}$-conjugate (for any $r$ ) to the normal form

$$
\varphi(y, \varepsilon) \frac{\partial}{\partial y} \pm \psi(y, \varepsilon) x \frac{\partial}{\partial x}
$$

It is easy to see that

$$
\varphi(y, \varepsilon)=\varepsilon \bar{\varphi}(y, \varepsilon)
$$

with $\bar{\varphi}(0,0) \neq 0$. In a sufficiently small neighbourhood of $(0,0), \bar{\varphi}$ is bounded by two positive constants $N, M$.

Hence, for a passage from $y_{0}$ to $y_{1}$, the transition time is calculated by

$$
T=\int_{0}^{\tilde{y}_{1}} \frac{d y}{\varepsilon \bar{\varphi}} \text {. }
$$

Therefore

$$
\frac{C}{\varepsilon} \leq T \leq \frac{D}{\varepsilon}
$$

for some positive constants $C, D$.

Of course in the simple situation we are dealing with we could just as well do this calculation directly without using a normal form; the current method is adapted to study more general problems.

4.2. Passage near the hyperbolic singularity. Let us start by changing the vector field $\bar{U}$ in the $x$-directional blown-up situation to the equivalent vector field

$$
U^{S}:\left\{\begin{array}{l}
\dot{u}=u \\
\dot{v}=\bar{\varepsilon}\left(v-\frac{1}{2}\right)^{-1}-2 v \\
\dot{\bar{\varepsilon}}=-\bar{\varepsilon}
\end{array}\right.
$$

and calculate the transit time of it between two points $\left(1, u_{0}\right)$ and $\left(\bar{\varepsilon}_{0}, 1\right)$ on respective sections $\{\bar{\varepsilon}=1\}$ and $\{u=1\}$. It is given by respectively $-\ln \left(u_{0}\right)$ or $-\ln \left(\bar{\varepsilon}_{0}\right)$ but we can also write it as $-\ln (\varepsilon)$ for $\varepsilon=u \bar{\varepsilon}$ since $u \bar{\varepsilon}=\varepsilon$ represents an invariant foliation. For the transit time in the original vector field 
we first observe that changing the transverse sections will not induce an essential difference and w.r.t. the given section we get the original transit time if we multiply $U^{S}$ by $u\left(v-\frac{1}{2}\right)$. For each $0<a<b$, with $a$ as small and $b$ as large as we want, and for $(u, v, \bar{\varepsilon})$ sufficiently near $(0,0,0)$ we get

$$
b u \bar{\varepsilon}<u\left(v-\frac{1}{2}\right)<a
$$

and hence

$$
\frac{1}{a}<\frac{1}{u\left(v-\frac{1}{2}\right)}<\frac{1}{b \varepsilon} .
$$

As such, the original transit time $T$ can be estimated as

$$
-\frac{1}{a} \ln (\varepsilon)<T<-\frac{1}{b} \frac{\ln (\varepsilon)}{\varepsilon} .
$$

4.3. Passage near a single semi-hyperbolic singularity. Starting from the blown up vector field in the $y$-direction, we perform a normal form calculation to obtain a relatively simple model equation. We simplify the computation by translating the singularity $(0,-\sqrt{2}, 0)$ to the origin with $v=\bar{v}-\sqrt{2}$ and by introducing the linear change of coordinates

$$
\left(\begin{array}{l}
x \\
y \\
z
\end{array}\right)=\left(\begin{array}{lll}
1 & 0 & 0 \\
0 & 1 & 1 \\
0 & 0 & 1
\end{array}\right)\left(\begin{array}{l}
u \\
\bar{v} \\
\bar{\varepsilon}
\end{array}\right) .
$$

The result is a new vector field $\tilde{X}^{\prime}(x, y, z)$ with linear part

$$
D \tilde{X}^{\prime} \mid(0,0,0)=\left(\begin{array}{ccc}
0 & 0 & 0 \\
0 & \sqrt{2} & 0 \\
0 & 0 & 0
\end{array}\right) \text {. }
$$

Again by Theorem 1 of [B], there exists a $C^{k}$-coordinate change, whose linear part is the identity, which transforms the vector field to

$$
\tilde{X}^{\prime \prime}\left\{\begin{array}{l}
\dot{x}=x z f(x, z) \\
\dot{y}=y g(x, z) \\
\dot{z}=-z^{2} f(x, z)
\end{array}\right.
$$

with $f(x, z)=1+\tilde{f}(x, z), \tilde{f}(0,0)=0, g(x, z)=\sqrt{2}-z+\tilde{g}(x, z), j_{1} \tilde{g}(0,0)$ $=0$, and leaving the foliation $x z=\varepsilon$ invariant. Because the variables are separated in (15), we can calculate the transit time by studying the easy equivalent two-dimensional model

$$
\left\{\begin{array}{l}
\dot{x}=x \\
\dot{z}=-z
\end{array}\right.
$$

Here, the time from $\left(x_{0}, 1\right)$ to $\left(1, z_{0}\right)$ is

$$
T_{S}=-\ln (\varepsilon) \text {. }
$$

Remark that we have rescaled the time by a factor $x$ (from the desingularization) and a factor $z$. Furthermore, we can choose our neighbourhood small enough so that, for any $\delta>0$,

$$
1-\delta \leq 1+\tilde{f}(x, z) \leq 1+\delta .
$$


So the original time $T(\varepsilon)$ has the property that

$$
\lim _{\varepsilon \rightarrow 0} \frac{\varepsilon T(\varepsilon)}{\ln (\varepsilon)}=-1
$$

which we write as

$$
T \sim-\frac{\ln (\varepsilon)}{\varepsilon} .
$$

Again, near such a semi-hyperbolic singularity we could easily obtain the same result from the expression of $\bar{U}^{\prime}$ itself without having to use a normal form theorem. However, we want to present it in this way in order to rely on techniques that can also be applied in more complicated situations.

The same observation can be made with respect to the next reasoning, where of course something has to be proved. Our approach, though not very long, is far too general for the example in consideration.

4.4. Passage near both semi-hyperbolic singularities. Finally, we consider the transition between the two semi-hyperbolic singularities. We calculate the passage time in three steps: the first, starting from the plane $\{z=1\}$ to $\{y=1\}$ in the normal form coordinates around the left singularity. The second part of the orbit is a regular passage between transverse sections $T_{1}$ and $T_{2}$. The third, between the planes $\{y=1\}$ and $\{x=1\}$ in the normal form coordinates of the right singularity. (See Figure 9.)

The contribution that any of the steps make to the total transit time (when $\varepsilon \rightarrow 0$ ) depends on the curve $x=\gamma(y)$, describing the initial conditions in the plane $\{z=1\}$. The sum of the contributions, however, reveals to be equivalent to $-\frac{\ln (\varepsilon)}{\varepsilon}$, for $\varepsilon \rightarrow 0$.

The regular passage can be described by the normal form $\frac{\partial}{\partial y}$ and does not contribute significantly to the transition asymptotics. If we start from a segment $L_{1}$ on the transversal section $T_{1}$, then the regular flow takes this to a segment $L_{2}$ on the transversal section $T_{2}$. Considered as a map between $L_{1}$ and $L_{2}$, the flow is a diffeomorphism. Hence, in normal form coordinates around the

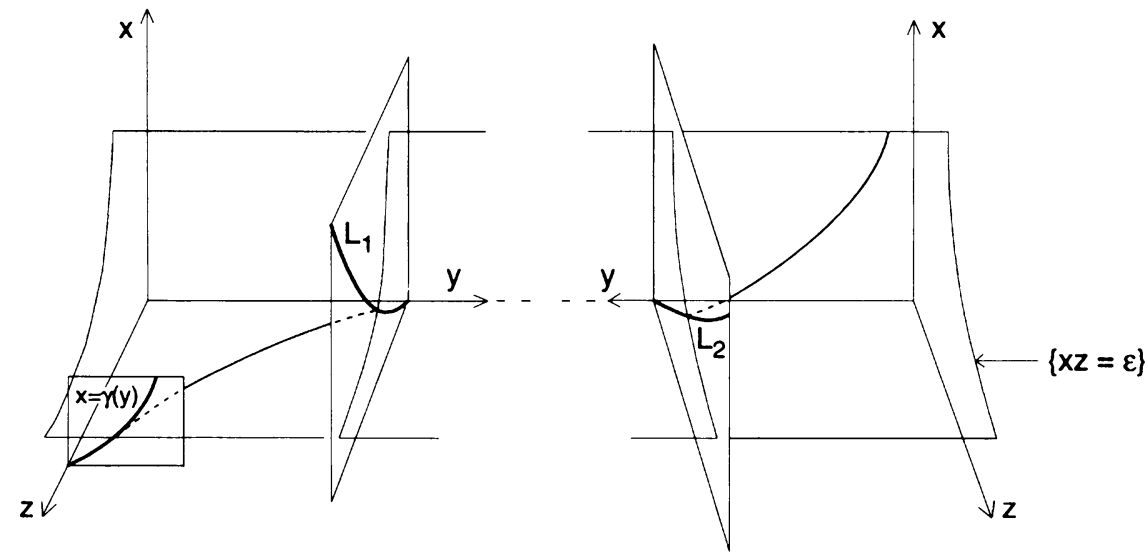

Figure 9. Passage near both semi-hyperbolic singularities. 
respective singularities, the order of contact between the segments and the $y z$ plane (or $x y$-plane) is preserved. In this way, a segment $L_{1}=\left\{x=a z^{k}+h . o . t.\right\}$ is mapped onto $L_{2}=\left\{x=b z^{k}+\right.$ h.o.t. $\}$.

In the normal form coordinates around any of the semi-hyperbolic singularities, the transit time asymptotics only depend on the $z$-coordinate of the intersection of the segment $L_{i}$ with the invariant plane $x z=\varepsilon$. Since the $\frac{\partial}{\partial x}$ and $\frac{\partial}{\partial z}$-components are the same in the normal forms of both singularities and since the $(x, z)$-variables are separated, the transit time is the same as with a single semi-hyperbolic singularity, up to the time needed for a passage between the sections $L_{1}$ and $L_{2}$ in any of the two normal form coordinates.

This last time can be calculated explicitly: for special segments $L_{1}=\{x=$ $\left.a z^{k}\right\}$ and $L_{2}=\left\{x=b z^{k}\right\}$ we have

$$
e^{T_{S}}=\left(\frac{a}{b}\right)^{\frac{1}{k+1}} \Rightarrow T_{S}=\frac{1}{k+1} \ln \left(\frac{a}{b}\right)
$$

thus constant.

For arbitrary non-flat contacts $L_{1}=\left\{x=a^{\prime} z^{k}+\right.$ h.o.t. $\}$ and $L_{2}=\{x=$ $b^{\prime} z^{k}+$ h.o.t. $\}$ we look for special sections $\left\{x=a z^{k}\right\}$ and $\left\{x=b z^{k}\right\}$ immediately above and below $L_{1} \cup L_{2}$ respectively. The transit time is then bounded by the transit time between these special segments, hence also bounded by a constant.

If the segments $L_{i}=\left(\varphi_{i}(z), z\right)$ have a flat contact with the $y z$-plane, we remark that we can find a constant $c$ such that, for any $k$, the segment $S_{k}=$ $\left\{x=c z^{k}\right\}$ lies above both $L_{1}$ and $L_{2}$. The transit time $T_{S}$ is then bounded by the transit from $\{z=1\}$ to any segment $S_{k}$, so

$$
T_{S}(k, \varepsilon)=\int_{z=1}^{z_{0}} \frac{d \zeta}{\zeta}=-\ln \left(z_{0}\right)=-\frac{1}{k+1} \ln (\varepsilon)+\frac{\lambda}{k+1}
$$

for any $k$, hence $T_{S}(k, \varepsilon) \rightarrow 0$, as $k \rightarrow+\infty$.

For segments that are tangent to the $x y$-plane, the calculations are the same if we work with the $x$-variable in the simple model.

As in the previous paragraph, we remark that we have scaled the transit time $T_{S}$ by a factor $\varepsilon$ and that, to compare it with the original time in (15), the neighbourhoods around the respective semi-hyperbolic singularities (in which the normal form coordinates hold) should be chosen small enough.

From (12), (14) and (16), we conclude that the minimal transit time of a solution passing left of the origin is equivalent to

$$
-\frac{\ln (\varepsilon)}{\varepsilon} \quad \text { as } \varepsilon \rightarrow 0
$$

\section{ASYMPTOTIC ANALYSIS AND CENTER MANIFOLDS}

In this section we show that classical outer expansion techniques geometrically amount to studying center manifold behaviour. The center manifold of the right semi-hyperbolic singularity in the $x$-directional blow-up can be calculated explicitly as $(\bar{\varepsilon}, w(\bar{\varepsilon}))$ up to any order from the equation

$$
\left\langle\left(1, \frac{d w}{d \bar{\varepsilon}}\right),\left(\bar{\varepsilon}-2\left(w(\bar{\varepsilon})+\frac{1}{2}\right) w(\bar{\varepsilon}), \bar{\varepsilon} w(\bar{\varepsilon})\right)\right\rangle=0
$$


Writing $w(\bar{\varepsilon})=\sum_{i=1}^{k} \alpha_{i} \bar{\varepsilon}^{i}$ this expression becomes

$$
\bar{\varepsilon}+\left(\sum_{i=1}^{k} \alpha_{i} \bar{\varepsilon}^{i}\right)\left(-1+\sum_{i=1}^{k}(i-2) \alpha_{i} \bar{\varepsilon}^{i}\right)=0
$$

As $k \rightarrow \infty$ we get

$$
\sum_{i=1}^{\infty}\left(\sum_{l=1}^{i-1} \alpha_{l} \alpha_{i-l}(l-2)-\alpha_{i}\right) \bar{\varepsilon}^{i}+\bar{\varepsilon}=0
$$

Solving this term per term is easy:

$$
\alpha_{1}=1 ; \alpha_{2}=-1 ; \alpha_{3}=1 ; \alpha_{4}=0 ; \alpha_{5}=-1 ; \alpha_{6}=-1 ; \text { etc. }
$$

with

$$
\alpha_{i}=\sum_{l=1}^{i-1} \alpha_{l} \alpha_{i-l}(l-2) \quad \text { for } i \geq 2 .
$$

In the original coordinates (which are blown up in the $x$-direction) this is formally expressed as

$$
w=v-\frac{1}{2}=\sum_{i=1}^{\infty} \alpha_{i} \bar{\varepsilon}^{i}
$$

hence

$$
u^{2} v=\frac{1}{2} u^{2}+\sum_{i=1}^{\infty} \alpha_{i} u^{2} \bar{\varepsilon}^{i}
$$

Using $\bar{\varepsilon}=\frac{\varepsilon}{u}$ we have

$$
y(x, \varepsilon)=\frac{1}{2} x^{2}+\alpha_{1} x \varepsilon+\sum_{i=2}^{\infty}\left[\sum_{l=1}^{i} \alpha_{l} \alpha_{i-l}(l-2)\right] x^{2-i} \varepsilon^{i}
$$

Now a formal expansion procedure in the original equation gives precisely the same result. Putting

$$
y(x, \varepsilon)=\sum_{i=0}^{\infty} y_{i}(x) \varepsilon^{i}
$$

and plugging this in the original system

$$
\left(y-\frac{1}{2} x^{2}\right) \frac{d y}{d x}=\varepsilon x^{2}
$$

gives us, choosing $y_{0}=\frac{1}{2} x^{2}$ (assuming that the orbit stays "close to the parabola")

$$
y_{1}(x)=\frac{1}{x} \text { and } \sum_{i=2}^{\infty}\left[\left(\sum_{l=0}^{i} y_{l}(x) y_{i-l}^{\prime}(x)\right)-\frac{1}{2} x^{2} y_{l}^{\prime}(x)\right] \varepsilon^{i}=0
$$

hence

$$
y_{i}(x)=-\frac{1}{y_{0}^{\prime}(x)} \sum_{l=0}^{i-1} y_{l}(x) y_{i-l}^{\prime}(x)
$$

It follows by induction that

$$
y_{k}(x)=\alpha_{k} x^{2-k}, \text { for all } k \geq 2 .
$$


Classically, one now calculates the time by substituting the expansion back into the equation:

$$
\begin{aligned}
\tilde{T} & =\int_{t_{0}}^{t_{1}} d t=\int_{x_{0}}^{x_{1}} \frac{\varepsilon d x}{y-\frac{1}{2} x^{2}}=\int_{x_{0}}^{x_{1}} \frac{d x}{x-\varepsilon+\varepsilon^{2}+\ldots} \\
& =\int_{x_{0}}^{x_{1}} \frac{d x}{x}+O(\varepsilon)
\end{aligned}
$$

If $x_{0}=O(\varepsilon)$ we find $\tilde{T}=O(-\ln (\varepsilon))$. Rescaling the time from (22) to our original vector field we obtain again $T=O\left(-\frac{\ln (\varepsilon)}{\varepsilon}\right)$.

To calculate the transition time asymptotics with the formal expansion technique, it is crucial to choose the right initial conditions in terms of $\varepsilon$. To see which is appropriate, we can look at surfaces $x=\varphi(\varepsilon)$ in the blown up situation. Choosing $x=\varepsilon$ (or equivalently $x=a \varepsilon$ with $a>0$ ) corresponds to starting from the plane $\bar{\varepsilon}=1$, respectively $\bar{\varepsilon}=a$ thus describing precisely the transition near the semi-hyperbolic singularity. The qualitative analysis performed in Sections 3 and 4 completely explains the reason why in the choice of initial conditions $x=\varphi(\varepsilon)$ we need to take $x \sim \varepsilon$ (or at least $x \sim a \varepsilon$ for some $a>0$ ) and not $x \sim \varepsilon^{\alpha}$ with $\alpha \neq 1$, in order to obtain the exact asymptotics of the transit time for $\varepsilon \rightarrow 0$.

However, the principal term in the asymptotic development of $T(\varepsilon)$ does clearly not indicate the limiting position (for $\varepsilon \rightarrow 0$ ) of the "minimal time" orbits. To find the limiting position (if it exists) we need to perform a more detailed analysis.

As a part of this let us observe that it is easy to show by classical asymptotic analysis that once passed near the degenerate singularity at the origin, (hence for $y>0$ ), the movement along the left branch of the parabola is faster than along the right one:

Using (22), we find

$$
T_{1}=\frac{1}{\varepsilon} \int_{\sqrt{2 y_{0}}}^{\sqrt{2 y_{1}}} \frac{d x}{x\left(1-\frac{\varepsilon}{x}+\frac{\varepsilon^{2}}{x^{2}}-\frac{\varepsilon^{4}}{x^{4}}+\ldots\right)}=\frac{1}{\varepsilon} \int_{\sqrt{2 y_{0}}}^{\sqrt{2 y_{1}}} \frac{d x}{x}\left(1+\frac{\varepsilon}{x}+O\left(\varepsilon^{3}\right)\right)
$$

and

$$
T_{2}=\frac{1}{\varepsilon} \int_{-\sqrt{2 y_{0}}}^{-\sqrt{2 y_{1}}} \frac{d x}{x\left(1+\frac{\varepsilon}{x}+\frac{\varepsilon^{2}}{x^{2}}+\frac{\varepsilon^{4}}{x^{4}}+\ldots\right)}=\frac{1}{\varepsilon} \int_{\sqrt{2 y_{0}}}^{\sqrt{2 y_{1}}} \frac{d x}{x}\left(1-\frac{\varepsilon}{x}+O\left(\varepsilon^{3}\right)\right)
$$

so $T_{2}<T_{1}$.

Along the branches it is also very easy to see that the calculation for center manifold reduction and formal expansion are exactly the same.

This time analysis seems to indicate that the limiting position (for $\varepsilon \rightarrow 0$ ) of the orbit between $y=y_{0}$ and $x=x_{0}$ could be like in the right picture of Figure 10.

This is also confirmed by numerical experiments we performed with the "dstool" package. A conclusive proof however, will require a more detailed analysis of the passage near the origin. 

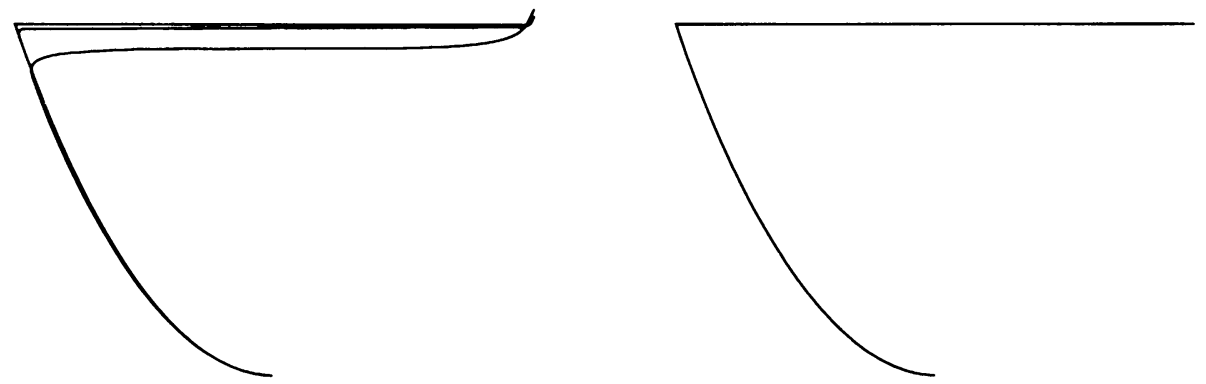

FIGURE 10. Minimal orbits (depending on $\varepsilon>0$ ) and their limit position.

\section{REFERENCES}

[AO] J.D. Allen and R.E. O'Malley, Jr., Singularly perturbed boundary value problems viewed in the Liénard plane, Asymptotic and Computational Analysis, (R. Wong, ed.), Lecture Notes in Pure and Applied Math., 124, Dekker, 1990, pp. 357-378.

[AP] D.K. Arrowsmith and C.M. Place, An introduction to dynamical systems, Cambridge University Press, 1990.

[B] P. Bonckaert, Partially hyperbolic fixed points with constraints, preprint (1993).

[Ca] J. Carr, Applications of centre manifold theory, Applied Mathematical Sciences 35, Springer, New York, 1981.

[CD] C. Chicone and F. Dumortier, Finiteness for critical periods of planar analytic vector fields, Nonlinear Analysis, Theory, Methods and Applications 20 (1993), 315-335.

[DeR] Z. Denkowska and R. Roussarie, A method of desingularization for analytic two-dimensional vector field families, Bol. Soc. Brasil. Mat. 22 (1991), 93-126.

[D] F. Dumortier, Techniques in the theory of local bifurcations: Blow-up, normal forms, nilpotent bifurcations, singular perturbations (notes written with B. Smits), Bifurcations of Periodic Orbits and Vector Fields (D. Schlomiuk, ed.), Nato ASI-series C408, 1993.

[DR] F. Dumortier and R. Roussarie, Canard cycles and center manifolds, preprint (1993).

[T1] F. Takens, Singularities of vector fields, Inst. Hautes Etudes Sci. Publ. Math. 43 (1973), 47-100.

[T2] F. Takens, Partially hyperbolic fixed points, Topology 10 (1971), 133-147.

Limburgs Universitair Centrum, Departement WNi, Universitaire Campus, B3590 DIEPENBEEK, BELGIUM

E-mail address: fdumorti@luc.ac.be

E-mail address: bsmits@luc.ac.be 